\title{
Neutrophil elastase and fetal fibronectin levels as predictors of single-birth prematurity
}

\author{
FANG AI, GUI-QING LI, JIANG JIANG and XU-DONG DONG \\ Department of Obstetrics, The First People's Hospital of Yunnan, Kunming, Yunnan 650032, P.R. China
}

Received July 22, 2014; Accepted March 25, 2015

DOI: $10.3892 /$ etm.2015.2508

\begin{abstract}
The aim of this study was to investigate the predictive values (PVs) of neutrophil elastase (NE) and fetal fibronectin (fFN) in cervical secretions for single-birth premature delivery. Samples of cervical secretions were obtained from 144 women with high-risk singleton pregnancies at 20-34 weeks' gestation and premature Creasy scores of $>12$ points for $\mathrm{NE}$ and fFN level testing, and the PVs of the two indicators for premature birth $(\mathrm{PB})$ were retrospectively analyzed. NE and fFN had high negative PVs (NPVs) for PB; the NPV of NE and fFN for delivery 7 days after detection was significantly higher than the positive PV $(\mathrm{P}<0.01)$. In addition, the sensitivity of the combined use of NE and fFN levels for PB prediction was high if both were present, and the $\mathrm{PB}$ rate of the double-positive group was higher than that of the single-positive group $(\mathrm{P}<0.01)$. Clinical intervention could turn the NE and fFN values negative in certain cases; in these cases, the PB rate was significantly lower than that in the sustained-positive group. In conclusion, NE and fFN in cervical secretions could be used as objective predictors of premature delivery, and their combined application could improve the prediction sensitivity. Effective clinical intervention could then reduce the incidence of PB.
\end{abstract}

\section{Introduction}

Premature birth (PB) is the primary cause of illness and mortality in perineonates, since $75-80 \%$ of cases of perineonatal mortality are associated with PB (1) and $50 \%$ of surviving perineonates have short- and long-term complications and sequelae (2). PB is a serious medical, social and economic problem (3). PB affects families and society in a negative manner, but prediction and prevention are the key to reducing its incidence and the incidence of perineonatal

Correspondence to: Professor Xu-Dong Dong, Department of Obstetrics, The First People's Hospital of Yunnan, 157 Jinbi Road, Kunming, Yunnan 650032, P.R. China

E-mail: dongxudongcn@126.com

Key words: premature birth, prediction, neutrophil elastase, fetal fibronectin, clinical intervention mortality; thus, accurate prediction methods are of paramount importance. Several studies regarding PB prediction have been conducted (4-6); however, most of them focused on fetal fibronectin (fFN) alone, lacked a multi-center design and had a limited study sample, and few reported on infection-related neutrophil elastase (NE). fFN is an isomer of fibronectin that has an important role in mediating the mutual adhesion and protection of the placenta and uterine decidua. The detection of fFN is important during pregnancy as it often indicates adhesion of the uterine decidua and placental villi, or damage to the fetal membrane $(7,8)$. NE belongs to the chymotrypsin superfamily of serine proteases, and hydrolyzes fibrous connective tissues outside cells during the inflammatory process; therefore, it is considered a significant predictor of inflammation $(9,10)$. This study analyzed NE and fFN in the cervicovaginal secretions as potential predictors of $\mathrm{PB}$ in singleton pregnancies and assessed the significance of the combination of the two indicators.

\section{Materials and methods}

Subjects. A total of 144 pregnant women who had been admitted to the First People's Hospital between June 2008 and March 2012, had a premature Creasy score (11) of $>12$ points and met the following criteria, were enrolled: Gestational age, 20-34 weeks; singleton pregnancy; intact fetal membrane and no vaginal bleeding; absence of other serious pregnancy complications; and no previous vaginal surgery, uterine contraction-inhibitors or sexual intercourse within $24 \mathrm{~h}$ of sampling. A dynamic monitoring method was used for repeated NE and fFN testing in the same patient at different gestational weeks. The aims of the repeated testing were to assess the feasibility of the two indicators for PB prediction and to observe the changes the indicators underwent following clinical interventions, as well as the effects of these changes on the pregnancy outcomes and outcomes of the observed pregnant women within and after the first 7 days of detection. This study was conducted in accordance with the Declaration of Helsinki and was approved by the Ethics Committee of the First People's Hospital of Yunnan Province (Kunming, China). Written informed consent was obtained from all participants.

Sampling methods of vaginal secretions. All pregnant women were examined in the bladder lithotomy position. A vaginal speculum was used to widen the vagina and expose the cervix, 
Table I. Basic information for the singleton pregnancies.

\begin{tabular}{lccccr}
\hline & & \multicolumn{2}{c}{ Gestation group } & & \\
\cline { 3 - 3 } Parameter & Total cases & $<34$ weeks & $\geq 34$ weeks & $\mathrm{t}$ & P-value \\
\hline Cases (n) & 133 & 37 & 96 & & \\
Mother's age (years) & $32.3 \pm 3.2$ & $32.5 \pm 4.7$ & $32.3 \pm 4.4$ & 0.183 & 0.852 \\
Pregnancy (n) & $1.0 \pm 1.1$ & $1.3 \pm 1.6$ & $0.9 \pm 0.9$ & -1.600 & 0.118 \\
Delivery (n) & $0.6 \pm 0.7$ & $0.7 \pm 1.0$ & $0.5 \pm 0.6$ & -1.301 & 0.197 \\
Gestational weeks at delivery & $35.8 \pm 3.9$ & $30.3 \pm 3.6$ & $37.7 \pm 1.5$ & 11.700 & $<0.001$ \\
\hline
\end{tabular}

Data are presented as the mean \pm standard deviation.

Table II. Association of NE and fFN with PB occurrence (delivery before the 34th gestational week) in single births.

\begin{tabular}{|c|c|c|c|c|c|c|c|}
\hline Indicator & Total (n) & $<34$ gestational weeks (n) & $\geq 34$ gestational weeks $(\mathrm{n})$ & SEN $(\%)$ & SPE (\%) & $\operatorname{PPV}(\%)$ & $\operatorname{NPV}(\%)$ \\
\hline $\mathrm{NE}$ & & & & 30.2 & 54.2 & 51.4 & 74.3 \\
\hline POS & 63 & 19 & 44 & & & & \\
\hline NEG & 70 & 18 & 52 & & & & \\
\hline fFN & & & & 86.5 & 62.5 & 47.0 & 92.3 \\
\hline POS & 68 & 32 & 36 & & & & \\
\hline NEG & 65 & 5 & 60 & & & & \\
\hline
\end{tabular}

Each patient was repeatedly tested for NE and fFN at different gestational weeks; a single positive result for NE or fFN in each patient is shown as a positive case in the table. NE, neutrophil elastase; fFN, fetal fibronectin; POS, positive; NEG, negative; SEN, sensitivity; SPE, specificity; PPV, positive predictive value; NPV, negative predictive value.

and a sterile test paper was then placed in the posterior part of the vaginal vault for $10-15 \mathrm{sec}$ to collect the cervicovaginal secretions.

Detection of fFN. Rapid fFN test paper (Adeza Biomedical, Sunnyvale, CA, USA) was used to determine the fFN level in the secretions by the clinician, strictly in accordance with the instructions of the sampling method. Following sampling, the top of the swab was placed in the buffer and fully mixed for 10-15 sec, after which the lower end of the fFN test paper was placed in the buffer for $10 \mathrm{~min}$ and then taken out to determine the results. An fFN level $\geq 50 \mu \mathrm{g} / \mathrm{ml}$ was considered positive and an $\mathrm{fFN}$ level $<50 \mu \mathrm{g} / \mathrm{ml}$ was considered negative.

Detection of NE. The NE detection kit was provided by the Affiliated Hospital of Japanese Kyorin University (Tokyo, Japan). The top of the sampled swab was placed in the reagent for $10 \mathrm{~min}$, transferred to the test paper and read within $15 \mathrm{~min}$. The simultaneous development of a reaction line and a quality control line indicated a positive result, whereas the development of a quality control line alone indicated a negative result.

Medical intervention. All NE- and fFN-positive pregnant women who exhibited symptoms of premature labor were admitted to the hospital and prescribed bed rest. Perineal scrubbing was performed, vaginal medication was administered, the frequency and intensity of uterine contractions were monitored, an adrenergic- $\beta$-receptor agonist or magnesium sulfate was administered to patients with frequent and intensive contractions to inhibit the contractions and glucocorticoids were timely used to promote fetal lung maturity. Following medication administration, NE and fFN were repeatedly assessed as markers of the PB treatment efficacy.

All NE- and fFN-negative pregnant women were administered only routine prenatal care without any other interventions, and their pregnancy outcomes were observed within and after 7 days of testing. NE and fFN values were also evaluated as prematurity predictors.

Statistical analysis. SPSS 11.5 statistical software (SPSS Inc., Chicago, IL, USA) was used for the statistical analysis, and the birth cases with positive or negative NE and fFN at the different gestational periods were expressed as percentages. The $\chi^{2}$ test was used to compare intergroup differences, and $\mathrm{P}<0.05$ was considered to indicate a statistically significant difference.

\section{Results}

Basic information. A total of 144 patients with singleton pregnancies were enrolled in and completed the study. Out of the 144 patients, 11 underwent scheduled cesarean section due to iatrogenic factors and thus their data were excluded from the 
Table III. Predictives values of NE and fFN for within-7-day delivery for single births.

\begin{tabular}{|c|c|c|c|c|c|c|c|}
\hline Indicator & Total (n) & $\begin{array}{c}\text { Parturition within } \\
7 \text { days of detection (n) }\end{array}$ & $\begin{array}{c}\text { Parturition after } \\
7 \text { days of detection (n) }\end{array}$ & $\operatorname{SEN}(\%)$ & $\operatorname{SPE}(\%)$ & PPV (\%) & $\operatorname{NPV}(\%)$ \\
\hline $\mathrm{NE}$ & & & & 27.2 & 86.9 & 11.2 & 95.1 \\
\hline POS & 89 & 10 & 79 & & & & \\
\hline NEG & 550 & 27 & 523 & & & & \\
\hline fFN & & & & 86.5 & 44.1 & 13.2 & 98.7 \\
\hline POS & 242 & 32 & 210 & & & & \\
\hline NEG & 397 & 5 & 392 & & & & \\
\hline
\end{tabular}

NE, neutrophil elastase; fFN, fetal fibronectin; SEN, sensitivity; SPE, specificity; POS, positive; NEG, negative; PPV, positive predictive value; NPV, negative predictive value.

Table IV. Association of NE and fFN with PB in single births.

\begin{tabular}{|c|c|c|c|c|}
\hline Parameter & $\mathrm{NE}^{+} \mathrm{fFN}^{+}$ & $\mathrm{NE}^{-} \mathrm{fFN}^{+}$ & $\mathrm{NE}^{+} \mathrm{fFN} \mathrm{N}^{-}$ & $\mathrm{NE}^{-} \mathrm{fFN} \mathrm{N}^{-}$ \\
\hline Parturition $<34$ gestational weeks, n $(\%)$ & $18(62.1)$ & $14(38.9)$ & $2(5.7)$ & $3(9.1)$ \\
\hline Parturition $\geq 34$ gestational weeks, n (\%) & $11(37.9)$ & $22(61.1)$ & $33(94.3)$ & $30(90.9)$ \\
\hline Total, n & 29 & 36 & 35 & 33 \\
\hline
\end{tabular}

NE, neutrophil elastase; fFN, fetal fibronectin.

Table V. Changes in NE status following clinical interventions and effect on the pregnancy outcomes in single births.

\begin{tabular}{|c|c|c|c|}
\hline Parameter & $\mathrm{NE}^{+} \rightarrow \mathrm{NE}^{+}$ & $\mathrm{NE}^{+} \rightarrow \mathrm{NE}^{-}$ & $\mathrm{NE}^{-} \rightarrow \mathrm{NE}^{-}$ \\
\hline Parturition $<34$ gestational weeks, n $(\%)$ & $8(88.9)$ & $11(20.4)$ & $18(25.7)$ \\
\hline Parturition $\geq 34$ gestational weeks, n (\%) & $1(11.1)$ & $43(79.6)$ & $52(74.3)$ \\
\hline Total, $\mathrm{n}$ & 9 & 54 & 70 \\
\hline
\end{tabular}

NE, neutrophil elastase; fFN, fetal fibronectin.

statistical analysis. Each patient was repeatedly tested for NE and fFN, undergoing 639 tests in total. Out of the 133 patients included in the analysis, 37 delivered before and 96 after gestational week 34 (27.8 vs. $72.2 \%$; Table I).

Correlation between $N E$ and $f F N$ levels and $P B$. The single detection results (Tables II and III) showed that the positive predictive value (PPV) of NE was slightly higher $(51.4 \%$ ) than that of fFN, but it was still not ideal as a stand-alone PB predictor (Table II). With regard to delivery within 7 days of detection, NE had a high negative predictive value (NPV) (95.1\%), particularly in cases of infection-induced PB, NE-negative patients and patients with a high likelihood of non-delivery within 7 days. These findings were similar to those for fFN, which had a higher NPV for delivery within 7 days (Table III).

Significance of combined NE-fFN prediction. When fFN was used alone, 32 cases of $\mathrm{PB}$ were predicted (sensitivity, 86.5\%), whereas 20 cases of PB were predicted when NE was used alone (sensitivity, 54.1\%). When fFN and NE were used together, 34 cases of PB were predicted (sensitivity, 91.9\%) but the specificity was decreased (specificity, 31.3\%). If the patients were both NE- and fFN-positive, the risk of PB increased considerably (Table IV).

Changes in NE and fFN and their effects on pregnancy outcomes. As shown in Table V, there were 9 cases of sustained positive NE following clinical intervention, and PB occurred in 8 out of these cases ( $88.9 \%$ ). Positive-to-negative NE transformation was noted in 54 cases, in 11 of which PB occurred (20.4\%). Out of the 70 cases of sustained negative NE, PB occurred in 18 cases $(25.7 \%)$. If the clinical interventions were effective, the transformation of NE-positive to NE-negative reduced the incidence of $\mathrm{PB}$, whereas a sustained positive NE greatly increased the chance of PB. Table VI shows that fFN underwent the same changes. Following clinical intervention, there were 42 cases of maintained positive fFN, and PB occurred in 31 of these cases $(73.8 \%)$. Twenty-four cases became fFN-negative, among which PB occurred only in 
Table VI. Changes in fFN status following clinical interventions and effect on the pregnancy outcomes in single births.

\begin{tabular}{lccc}
\hline Parameter & $\mathrm{fFN}^{+} \rightarrow \mathrm{fFN}^{+}$ & $\mathrm{fFN}^{+} \rightarrow \mathrm{fFN}^{-}$ & $\mathrm{fFN}^{-} \rightarrow \mathrm{fFN}^{-}$ \\
\hline Parturition $<34$ gestational weeks, $\mathrm{n}(\%)$ & $31(73.8)$ & $1(4.2)$ & $5(7.5)$ \\
Parturition $\geq 34$ gestational weeks, $\mathrm{n}(\%)$ & $11(26.2)$ & $23(95.8)$ & $62(92.5)$ \\
Total, $\mathrm{n}$ & 42 & 24 & 67 \\
\hline
\end{tabular}

NE, neutrophil elastase; fFN, fetal fibronectin.

1 case $(4.2 \%)$. fFN maintained its positivity in 67 cases, in 5 of which PB occurred $(7.5 \%)$.

In summary, the NE and fFN status may become negative following effective clinical intervention, leading to a decrease in the likelihood of PB. In cases of sustained NE and fFN positivity, the likelihood of PB can increase, and these patients may require aggressive treatment, such as fetal lung maturity promotion, to increase the fetal survival rate.

\section{Discussion}

PB is the main cause of morbidity and mortality in perineonates and its serious consequences constitute an indisputable social and family burden. Although perinatal care and obstetric interventions have been significantly strengthened over time, the occurrence of PB has not notably declined; it has instead increased annually, making $\mathrm{PB}$ a considerable focus of attention (12). The effects of interventions for PB are often unsatisfactory due to delays in their administration (13); therefore, identifying effective predictor(s) of PB that will enable timely intervention is of great clinical importance. Numerous studies have indicated that $50 \%$ of PB cases are infection-related $(14,15)$ and that subclinical infection, in late pregnancy in particular, is closely associated with PB (16). fFN and NE were the two most important indicators of inflammation in these studies.

It is known that fFN, an isomer of fibronectin, is produced by chorionic trophoblast cells (17) and plays an important role in mediating the mutual adhesion and protection of the placenta and uterine decidua. In the early stage of a normal pregnancy, as a result of the lack of fusion between the chorion and decidua capsularis in the parietal decidua of the uterine wall, low levels of fFN can leak out. Increasing levels of fFN prior to delivery often result from uterine contraction or mechanical trauma caused by the separation of the chorionic uterine walls (18). In addition, the detection of fFN during pregnancy often indicates adhesion of the uterine decidua and placental villi or damage to the fetal membrane; the main reason for its detection is damage caused by inflammatory material to the villus tissues (19).

Lockwood first reported the role of fFN in PB prediction in 1991 (20); thereafter, it was proposed in a number of studies that fFN in cervicovaginal secretions in the advanced stage of pregnancy was a powerful predictive marker for spontaneous PB $(21,22)$. The American Congress of Obstetricians and Gynecologists has recommended fFN detection as an important step in PB diagnosis (23). In the present study, the sensitivity, specificity, PPV and NPV of fFN for PB were 86.5,
$62.5,47.0$ and $92.3 \%$, respectively, indicating that fFN was an effective predictor of PB. The high NPV of fFN indicated that negative fFN results are associated with a low possibility of $\mathrm{PB}$ occurrence for pregnant women with PB symptoms, a finding that was consistent with that of Roman et al (24). With regard to $\mathrm{PB}$ prediction, estimating the likelihood of delivery within 7 days of prediction could have considerable clinical value, and could lead to a reduction in the incidence of PB through timely clinical intervention. The findings of the present study showed that the sensitivity of fFN for predicting within-1-week delivery was $86.5 \%$, with a specificity of $44.1 \%$, a PPV of 13.2 and an NPV of $98.7 \%$, thus leading to the conclusion that fFN is an effective predictor of within-1-week delivery.

Clinical studies have found that vaginal and cervical infection-induced ascending intra-amniotic cavity infection is an important cause of PB (25). When the cervix is infected, it can cause the local invasion and large accumulation of inflammatory cells and the release of inflammatory cytokines under the stimulation of inflammatory factors, followed by neutrophil activation and degranulation with NE release $(26,27)$. NE belongs to the chymotrypsin superfamily of serine proteases, which mainly hydrolyzes proteins through phagocytosis within cells and hydrolyzes fibrous connective tissues outside cells during the inflammatory process. The extracellular hydrolytic objects of NE include elastin, proteoglycans, fibronectin, laminin and collagens I, II, III and IV $(26,28)$.

Certain studies have shown that, in pregnant women with premature membrane rupture and threatened premature labor, the concentration of NE in the amniotic fluid is markedly increased, suggesting that $\mathrm{NE}$ is a useful indicator of amniotic infection and could be used to predict PB $(29,30)$. Nakai et al (31) found that the NPV of NE for PB was high at $92 \%$, which suggested that the granulocyte-released NE in the cervical secretions could be used as an independent predictor of delivery before gestational week 34. In the present study, 144 women with high-risk pregnancies underwent cervical secretion testing (NE detection) between the 20th and 34th gestational weeks, and the pregnancy outcomes within and after 7 days of detection were observed. The results revealed that NE had a slightly higher PPV than fFN (51.4\%) but its use as a single predictor was still not ideal. Taking only the deliveries that occurred within 7 days into consideration, NE exhibited a high NPV (95.1\%); thus, if the patient was NE-negative, the likelihood of non-delivery within 7 days would be high, similar to that in fFN-negative patients, as fFN negativity also had a high NPV for delivery within 7 days. These results were similar to those of the study by Nakai et al (31). 
PB does not have a single cause and an increasing number of scholars have come to advocate the combined application of indicators in order to improve their predictive values and overcome the limitations of a single predictor $(32,33)$. To confirm whether the combination of a number of indicators would prove more effective than the use of an individual indicator, this study analyzed the combined use of NE and fFN. fFN alone predicted $\mathrm{PB}$ in 32 cases (sensitivity, 86.5\%), NE alone predicted PB in 20 cases (sensitivity, 54.1\%) and $\mathrm{NE}$ and fFN in combination predicted PB in 34 cases (sensitivity, $91.9 \%$ ) but with a decreased specificity (31.3\%). Furthermore, it was observed that the risk of $\mathrm{PB}$ was significantly increased in the NE- and fFN-positive patients. Generally, a high NPV can be used to exclude the pseudosymptoms of PB and limit the administration of uterine contraction-inhibiting drugs, and a low PPV and sensitivity can also limit the clinical use of these drugs. It is therefore believed that the combination of several indicators could improve the prediction reliability and, in particular, that combined screening for $\mathrm{NE}$ and $\mathrm{fFN}$ would be more effective than screening for a single index.

The aim of PB prediction is to effectively prevent PB. In the present study, all patients received clinical interventions, including basic treatment, uterine contraction inhibitors and topical anti-infective therapy. Following clinical intervention, the NE-positive status was maintained in 9 cases, and PB occurred in 8 of these cases $(88.9 \%)$. There were 43 cases of NE-positive to NE-negative transformation, and PB occurred in 11 of these cases $(20.4 \%)$. Thus, the transformation to NE-negative status following effective clinical intervention may lead to the reduction of PB incidence, whereas the sustained NE-positive status following effective clinical intervention indicates a considerably increased risk of PB. The same changes were observed with fFN transformation. Following clinical intervention, fFN positivity was maintained in 42 cases, in 31 (73.8\%) of which PB occurred, and 24 cases of fFN-positive to fFN-negative transformation were observed, in only 1 (4.2\%) of which PB occurred.

As mentioned above, NE and $\mathrm{fFN}$ statuses may turn negative following effective clinical intervention, leading to a decrease in the likelihood of PB. Sustained positivity, by contrast, is indicative of an increased possibility of PB occurrence, which may necessitate aggressive treatment, such as fetal lung maturity promotion, to increase the survival rate of premature infants.

PB prediction previously relied on non-specific and non-sensitive indicators, such as obstetric history and clinical symptoms, which increased the possibility of delayed prediction and treatment. The early detection of PB and timely intervention are therefore the key to preventing PB and improving adverse pregnancy outcomes. Preventing PB and developing a non-invasive prediction method should be the priority of obstetric workers. In conclusion, the present study monitored levels of fFN and NE within cervicovaginal secretions and found that the combination of these two indicators could improve the sensitivity of PB prediction, thus providing a novel strategy for the prediction of $\mathrm{PB}$.

\section{Acknowledgements}

This study was supported by the Combined Applied-Scientific Research Projects of the Department of Science and
Technology of Kunming Medical College, Kunming, Yunnan (grant no. 2007C0035R) and the projects of Backup Talented Young Academic Leader, Yunnan (grant no. 2008PY045).

\section{References}

1. Iams JD, Goldenberg RL, Mercer BM, et al; National Institute of Child Health and Human Development Maternal-Fetal Medicine Units Network: The preterm prediction study: Can low-risk women destined for spontaneous preterm birth be identified? Am J Obstet Gynecol 184: 652-655, 2001.

2. Russo A, McCready M, Torres L, et al: Reducing hypothermia in preterm infants following delivery. Pediatrics 133: e1055-e1062, 2014.

3. Pappas A, Kendrick DE, Shankaran S, et al; Eunice Kennedy Shriver National Institute of Child Health and Human Development Neonatal Research Network: Chorioamnionitis and early childhood outcomes among extremely low-gestational-age neonates. JAMA Pediatr 168: 137-147, 2014.

4. Borg F, Gravino G, Schembri-Wismayer P and Calleja-Agius J: Prediction of preterm birth. Minerva Ginecol 65: 345-360, 2013.

5. Bolt LA, Morrison K and Shennan AH: The use of fetal fibronectin testing and cervical length measurement in the prediction of delivery of triplet pregnancies. Eur J Obstet Gynecol Reprod Biol 164: 236-237, 2012.

6. Bastek JA, Hirshberg A, Chandrasekaran S, et al: Biomarkers and cervical length to predict spontaneous preterm birth in asymptomatic high-risk women. Obstet Gynecol 122: 283-289, 2013.

7. Zhang S, Fu S, Liu Y, et al: Predictive value of fetal fibronectin on the embryonic loss of patients with recurrent spontaneous abortion in early pregnancy. Clin Exp Obstet Gynecol 41: 380-383, 2014.

8. Foster $\mathrm{C}$ and Shennan AH: Fetal fibronectin as a biomarker of preterm labor: A review of the literature and advances in its clinical use. Biomark Med 8: 471-484, 2014.

9. Ikeda S, Kihita K, Yokoi A, Tamakoshi K, Miyazaki K and Furuhashi M: The levels of the neutrophil elastase in the amniotic fluid of pregnant women whose infants develop bronchopulmonary dysplasia. J Matern Fetal Neonatal Med 28: 479-483, 2015.

10. Le Gars M, Descamps D, Roussel D, et al: Neutophil elastase degrades cystic fibrosis transmembrane conductance regulator via calpains and disables channel function in vitro and in vivo. Am J Respir Crit Care Med 187: 170-179, 2013.

11. Honest H, Bachmann LM, Sundaram R, Gupta JK, Kleijnen J and Khan KS: The accuracy of risk scores in predicting preterm birth - a systematic review. J Obstet Gynaecol 24: 343-359, 2004.

12. Barrios YV, Sanchez SE, Qiu C, Gelaye B and Williams MA: Risk of spontaneous preterm birth in relation to maternal experience of serious life events during pregnancy. Int J Womens Health 6: 249-257, 2014.

13. van Vliet EO, Boormans EM, de Lange TS, Mol BW and Oudijk MA: Preterm labor: Current pharmacotherapy options for tocolysis. Expert Opin Pharmacother 15: 787-797, 2014.

14. Klein LL and Gibbs RS: Infection and preterm birth. Obstet Gynecol Clin North Am 32: 397-410, 2005.

15. Newton ER: Preterm labor, preterm premature rupture of membranes and chorioamnionitis. Clin Perinatol 32: 571-600, 2005.

16. Park CW, Yoon BH, Kim SM, Park JS and Jun JK: The frequency and clinical significance of intra-amniotic inflammation defined as an elevated amniotic fluid matrix metalloproteinase- 8 in patients with preterm labor and low amniotic fluid white blood cell counts. Obstet Gynecol Sci 56: 167-175, 2013.

17. Fjaertoft G, Håkansson L, Foucard T, Ewald U and Venge P: CD64 (Fcgamma receptor I) cell surface expression on maturing neutrophils from preterm and term newborn infants. Acta Paediatr 94: 295-302, 2005.

18. Rossel Goffeng A, Holst E, Milsom I, Lindstedt G, Lundberg PA and Andersch B: Fetal fibronectin and microorganisms in vaginal fluid of healthy pregnant women. Acta Obstet Gynecol Scand 75: 520-525, 1996.

19. Yoon BH, Romero R, Moon JB, Oh SY, Han SY, Kim JC and Shim SS: The frequency and clinical significance of intra-amniotic inflammation in patients with a positive cervical fetal fibronectin. Am J Obstet Gynecol 185: 1137-1142, 2001. 
20. Lockwood CJ, Senyei AE, Dische MR, Casal D, Shah KD, Thung SN, Jones L, Deligdisch L and Garite TJ: Fetal fibronectin in cervical and vaginal secretions as a predictor of preterm delivery. N Engl J Med 325: 669-674, 1991.

21. Conde-Agudelo A and Romero R: Cervicovaginal fetal fibronectin for the prediction of spontaneous preterm birth in multiple pregnancies: A systematic review and meta-analysis. J Matern Fetal Neonatal Med 23: 1365-1376, 2010.

22. Abdelazim IA: Fetal fibronectin [Quick Check fFN test(®)] for detection of premature rupture of fetal membranes. Arch Gynecol Obstet 287: 205-210, 2013.

23. Peláez LM, Fox NS and Chasen ST: Negative fetal fibronectin: Who is still treating for threatened preterm labor and does it help? J Perinat Med 36: 202-205, 2008.

24. Roman AS, Rebarber A, Lipkind H, Mulholland J, Minior V and Roshan D: Vaginal fetal fibronectin as a predictor of spontaneous preterm delivery after multifetal pregnancy reduction. Am J Obstet Gynecol 190: 142-146, 2004

25. Silver BJ, Guy RJ, Kaldor JM, Jamil MS and Rumbold AR: Trichomonas vaginalis as a cause of perinatal morbidity: A systematic review and meta-analysis. Sex Transm Dis 41: 369-376, 2014.

26. Faye-Petersen OM: The placenta in preterm birth. J Clin Pathol 61: 1261-1275, 2008.

27. Czerwińska ME, Kiss AK and Naruszewicz M: Inhibition of human neutrophils NEP activity, CD11b/CD18 expression and elastase release by 3,4-dihydroxyphenylethanol-elenolic acid dialdehyde, oleacein. Food Chem 153: 1-8, 2014.
28. Krupa FG, Faltin D, Cecatti JG, Surita FG and Souza JP: Predictors of preterm birth. Int J Gynaecol Obstet 94: 5-11, 2006.

29. Czajka R, Kwiatkowski S, Chlubek D, Dołegowska B, Torbé A and Rzepka R: The assessment of neutrophil elastase measurements usefulness in pregnant women with premature rupture of fetal membranes and chorioamnionitis suspicion. Ginekol Pol 80: 19-24, 2009 (In Polish).

30. Kidokoro K, Furuhashi M, Kuno N and Ishikawa K: Amniotic fluid neutrophil elastase and lactate dehydrogenase: Association with histologic chorioamnionitis. Acta Obstet Gynecol Scand 85: 669-674, 2006

31. Nakai A, Taniuchi Y, Miyake H, Nakai M, Yokota A and Takeshita T: Increased level of granulocyte elastase in cervical secretion is an independent predictive factor for preterm delivery. Gynecol Obstet Invest 60: 87-91, 2005.

32. Morken NH, Källen K and Jacobsson B: Predicting risk of spontaneous preterm delivery in women with a singleton pregnancy. Paediatr Perinat Epidemiol 28: 11-22, 2014.

33. Sananes N, Meyer N, Gaudineau A, et al: Prediction of spontaneous preterm delivery in the first trimester of pregnancy. Eur J Obstet Gynecol Reprod Biol 171: 18-22, 2013. 